\title{
Indirect Reduction and Intramedullary Pinning in Severely Displaced Radial Neck Fractures in Children
}

\author{
Ashish Devgan, Raj Singh, Suresh Kumar, Vinit Verma, N. K. Magu, R. C. Siwach \\ Department of Orthopaedics, Pt. B.D.Sharma University of Health Sciences, Rohtak, India. \\ Email: ashishdevgan@gmail.com
}

Received August $18^{\text {th }}$, 2010; revised January $4^{\text {th }}, 2011$; accepted January $11^{\text {th }}, 2011$.

\begin{abstract}
Fractures of the radial neck account for 5\% - 10\% of traumatic lesions of the elbow in the child. Conservative method of reduction may fail to reduce severely displaced fractures. Open reduction of severely displaced radial neck fractures is associated with a high incidence of complications. The present study describes the results achieved after closed and indirect reduction of the severely displaced radial head using a 2-mm K-wire passed intramedullary from near the radial styloid. The bent tip of the K-wire was used to manipulate the displaced head back to the shaft without opening the fracture site. The indirect reduction of the radial head using bent intramedullary K-wire is a simple, minimally invasive method, easy to perform, and obtains encouraging results with few complications. Intramedullary pin also achieves fixation of the radial head after reduction and prevents its secondary displacement.
\end{abstract}

Keywords: Intramedullary Pinning, Radial Neck Fracture

\section{Introduction}

Fractures of the radial neck is a comparatively rare injury and accounts for $5 \%-10 \%$ of traumatic lesions of the elbow in the child [1]. They usually occur between 5 and 13 years of age [2]. Fractures of the neck of the radius in children are an unsolved and difficult problem. It remains a source of considerable controversy and has many possible complications that are usually hazardous for the elbow function. The complex anatomy of the elbow and its vascular blood supply also makes treatment challenging. The radial head and its blood supply can be damaged by the original trauma and/or by surgical trauma, such as open reduction or forceful manipulations. The choice of treatment is determined by the degree of radial head angulation, which also affects the long-term results. Severe angulation and translation increases the risk of complications, mainly malunion, avascular necrosis of the head, cubitus valgus and stiffness of the joint. Conservative treatment in the form of closed reduction and plaster immobilization is usually successful for the nondisplaced and minimally displaced fractures while in severely displaced fractures of angulation more than 45 degrees, open reduction is warranted if closed reduction fails [3-7]. Conservative treatment in the form of closed reduction and plaster immobilization is unable to reduce severe displacements, resulting in malunion [1]. Open reduction of severely displaced radial neck fractures is associated with a high incidence of complications [8]. Percutaneous pinning of displaced fragment has been associated with serious complications like wire breakage and nerve injury [6]. In 1980, Metaizeau proposed intramedullary nailing as a surgical option for the treatment of radial neck fractures. The main advantage of intramedullary nailing is that it simultaneously allows accurate and stable reduction without disturbing the blood supply. The present study reports the results of 12 cases of severely displaced radial neck fractures managed by less invasive technique of intramedullary pinning.

\section{Material and Methods}

This paper is a prospective study of 12 children of age ranging from 7 to 9 years of which 9 were males and 3 females. All had history of fall on outstretched hand with the elbow extended. There were 8 right and 4 left limbs that were involved. Physical examination revealed swelling and tenderness over the lateral aspect of elbow. There was no distal neurovascular deficit. Anteroposterior (AP) and lateral radiographs of the elbow showed fracture of 
radial neck with more than 60 degree of angulation (type III fracture O'Brien classification) [9] and more than 50\% translation (see Figures 1 and 2) in all cases. The angulation of the fracture was measured as the angle between a line perpendicular to the articular surface of the radial head with a line down the shaft of proximal radius. The translation of the proximal fragment was measured in terms of percentage, where the width of the uncovered radial metaphyses was divided by the total width of the metaphyses (multiplied by 100).

A gentle manipulation was attempted under sedation to achieve closed reduction using Patterson's technique [10]. But closed reduction failed to reduce the fracture. Then, closed reduction was attempted under general anaesthesia in supine position using intramedullary K-wire under image intensifier control. A 1-cm skin incision was made along the lateral border of the distal radial metaphysis
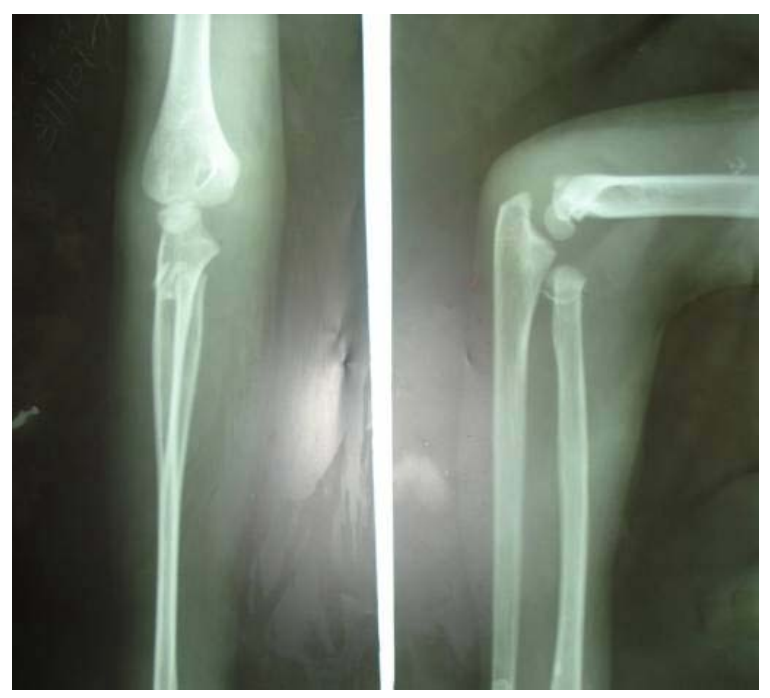

Figure 1. Preoperative radiograph (Case 1).

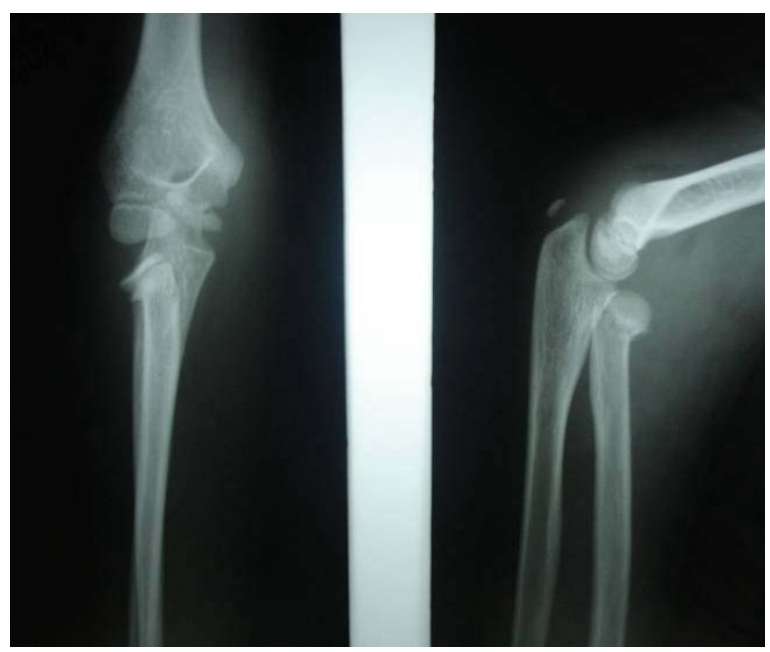

Figure 2. Preoperative radiograph (Case 2). about $2 \mathrm{~cm}$ proximal to the epiphyseal plate. Soft tissues were dissected with scissors, avoiding the superficial branch of radial nerve. A bone awl was used to make entry point for K-wire insertion about $2 \mathrm{~cm}$ proximal to epiphyseal plate (see Figure 3).

A 2-mm diameter K-wire was contoured and the last 3 $\mathrm{mm}$ portion of it was bent approximately 30 degrees. The K-wire was introduced into the medullary canal of the radius and was hammered slowly upwards till its point reached the inferior aspect of the fracture. Rotatory movements with use of $\mathrm{T}$ handle and minimal force made the insertion easy. Wire was advanced by gentle taps of the hammer till its point fixed in the epiphysis. Epiphysis was then elevated until it was repositioned under the lateral condyle. The remaining lateral shift was corrected by turning the K-wire around its long axis through 180 degrees which resulted in the facing of the point of $\mathrm{K}$ wire towards ulna carrying with it and repositioning the displaced fragment (see Figure 4). After achieving satisfactory reduction, the lower metaphyseal end of the Kwire was cut and skin closed. The elbow was immobilized in flexion in neutral position for 2 weeks, after which gentle active range-of-motion exercises were begun. The wire was removed after fracture union at an average of 10 weeks after surgery.

\section{Results}

Patients did not develop any postoperative complication. At final review (26 months), all the fractures had proceeded to union (see Figures 5 and 6). Radiographs did not reveal any complication including avascular necrosis of radial head, premature fusion of proximal radial epiphysis, radioulnar synostosis, or myositis ossificans and all children achieved normal carrying angle. Patient did not develop any loss of joint motion, instability, or deformity of elbow till last follow-up.
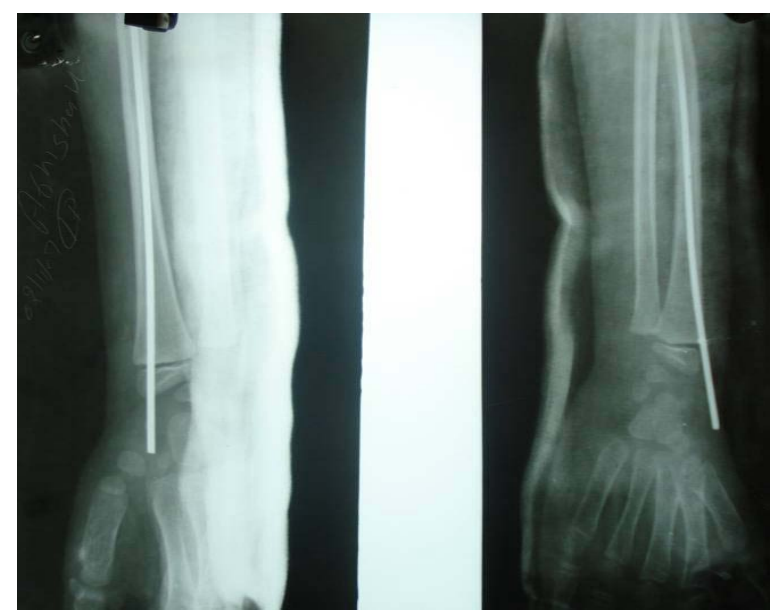

Figure 3. Radiograph showing entry portal $2 \mathrm{~cm}$ proximal to epipyseal plate. 


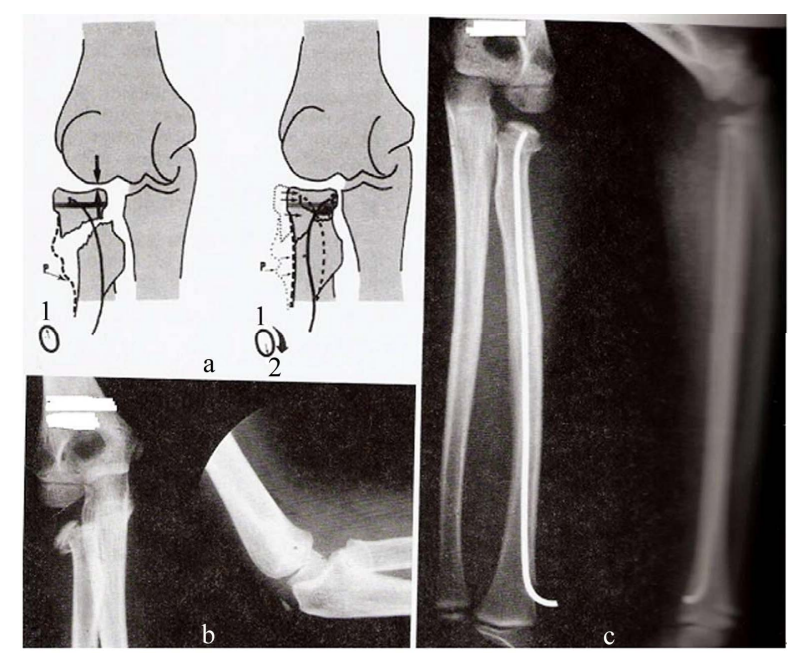

Figure 4. Joy stick maneuver by the bent tip of intramedullary K-wire, reduces the fracture.
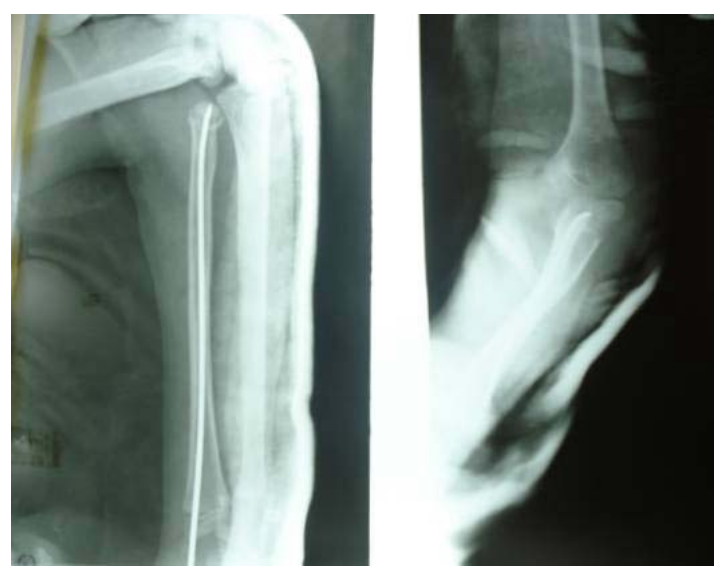

Figure 5. Postoperative radiograph showing fracture union (Case 1).

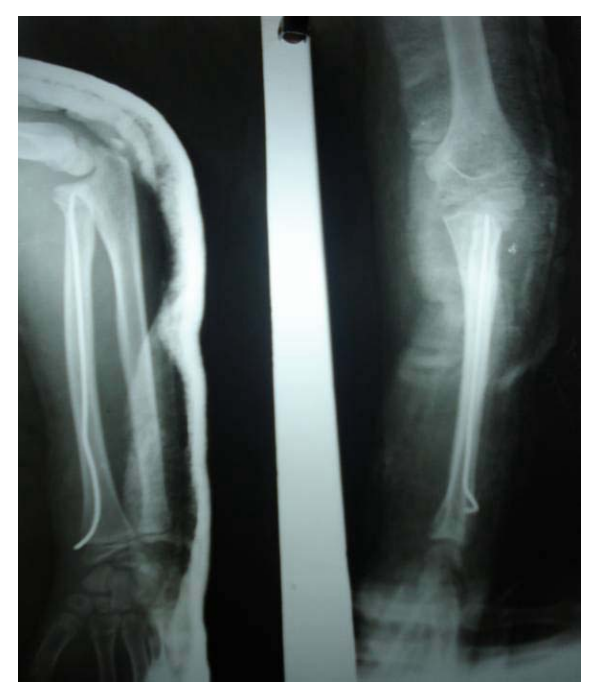

Figure 6. Postoperative radiograph showing well aligned fracture union (Case 2).

\section{Discussion}

Radial neck fractures are not uncommon and have many possible complications that are frequently detrimental to joint function. The whole radial head epiphysis is surrounded by articular cartilage. So the vessels can only enter the bone at insertion of periosteum [1]. Fracture of the neck disrupts part of the periosteum with its vessels, more so in severely displaced fractures. Open reduction of the fracture can further damage the vascularity of the radial head [11]. The high complication rate after open reduction and the poor functional results and inconvenience for the pediatric patients makes conservative treatment of radial neck fractures in children preferable $[11,12]$. An attempt at closed reduction is recommended for fractures that are angulated more than 30 degrees $[4,5$, 8]. Closed reduction may fail in severely displaced fractures [5-7]. Newer methods of treatment continue to develop to treat these difficult, severely displaced fractures $[13,14]$. Dawson and Inostroza reported a technique of reduction with arthroscopic assistance and percutaneous stabilization that allowed better intra-articular evaluation and reduction control with minimal soft tissue trauma [13]. Percutaneous methods of reduction have been developed in an effort to avoid the higher incidence of complications after open reduction. This technique involves direct pressure on the proximal fragment with a Steinmann pin or K-wire that is introduced percutaneously or through a small stab wound [15-17]. This technique of percutaneous “joystick" reduction can injure the posterior interosseous nerve $[8,10]$.

Intramedullary manipulation of the radial head is another method of percutaneous, indirect reduction of the fracture. This method of indirect reduction does not jeopardize further the vascularity of the radial head which can occur in open reduction. Lateral condyle acts as a buffer to prevent overcorrection in long axis whereas tension produced in the lateral intact periosteum prevents medial overcorrection (see Figure 4).

We were successful in closed reduction of the fracture using this technique in all cases. When closed reduction with intramedullary pin fails, an additional joystick technique using a percutaneous wire can be used to fully reduce the radial head $[1,8,18]$. However, if tilt is more than 80 degrees, it is sometimes impossible to reach the epiphysis with the point of wire . Other authors have also reported satisfactory results of closed reduction and intramedullary pinning $[12,19]$. We did not observe any complication in the 12 cases up to the longest follow-up of 26 months. However, complications reported after intramedullary pinning include avascular necrosis of radial head, loss of joint motion, particularly of pronation/supination, and anterior epiphysiodesis [12,19]. Pra- 
thapkumar et al. [20] reported 14 children treated by indirect reduction and fixation using an elastic stable intramedullary nail. The patients were followed up for a mean of 28 months. One developed asymptomatic avascular necrosis. The rest had excellent results. One had a neuropraxia of the posterior interosseous nerve, which recovered within six weeks. Garcia-Alvarez et al. [21] reported a comparative retrospective analysis of 20 displaced radial neck fractures with more than 30 degrees of tilt treated with closed intramedullary pinning and arthrotomy and pinning. The former technique produced better results and fewer complications than arthrotomy and pinning. They also observed a relationship between residual final tilt and poor functional results.

Our study has limitations by the small number of patients. By this study we recommend closed reduction using intramedullary pinning because it is a simple, indirect, minimally invasive method, easy to perform, and obtains exciting results with few complications. Intramedullary pin also achieves fixation of the radial head after reduction and prevents its secondary displacement. Open reduction should be reserved for those fractures irreducible by the percutaneous methods.

\section{REFERENCES}

[1] J. P. Metaizeau, "Reduction and Osteosynthesis of Radial Neck Fractures in Children by Centromedullary Pinning," Injury, Vol. 36, Suppl. 1, 2005, pp. 75-77. doi:10.1016/j.injury.2004.12.016

[2] J. P. Dormans and M. Rang, "Fractures of the Olecranon and Radial Neck in Children," Orthopedic Clinics of North America, Vol. 21, No. 2, 1990, pp. 257-268.

[3] G. Okcu, "Treatment Options for Radial Neck Fractures in Children,” Pediatric Health, Vol. 3, No. 1, 2009, pp. 67-73. doi:10.2217/17455111.3.1.67

[4] J. E. Tibone and M. Stoltz, "Fracture of the Radial Head and Neck in Children,” The Journal of Bone and Joint Surgery, Vol. 63, No. 1, 1981, pp. 100-106.

[5] E. L. Steinberg, D. Golumb, R. Salam and S. Weintroub, "Radial Head and Neck Fracture in Children," Journal of Pediatric Orthopaedics, Vol. 8, 1988, p. 35.

[6] J. V. Fowles and M. T. Kasab, “Observations Concerning Radial Neck fractures in Children,” Journal of Pediatric Orthopaedics, Vol. 6, 1986, pp. 51-53.

[7] J. V. Pseudo, J. Aracil and M. Barcelo, "Leverage Method in Displaced Fracture of Radial Neck," Clinical Orthopaedics, Vol. 169, 1982, pp. 215-217.

[8] T. E. Radomisli and A. L. Rosen, “Controversies Regarding Radial Neck Fractures in Children,” Clinical Orthopaedics and Related Research, Vol. 353, 1998, pp. 30-39.

[9] P. I. O’Brien, "Injuries Involving the Proximal Radial Epiphysis,” Clinical Orthopaedics and Related Research, Vol. 41, 1965, pp. 51-58.
[10] J. A. Herring, “Upper Extremity Injuries,” Tachdjian's Pediatric Orthopaedics, 3rd Edition, Saunders, Philadelphia, 2002, pp. 2194-2200.

[11] A. K. Vocke and L. Von Laer, "Displaced Fractures of the Radial Neck in Children: Long-Term Results and Prognosis of Conservative Treatment," Journal of Pediatric Orthopaedics B, Vol. 7, No. 3, 1998, pp. 217-222. doi:10.1097/01202412-199807000-00007

[12] P. P. Schmittenbecher, B. Haevernick, A. Herold, P. Knorr and E. Schmid, "Treatment Decision, Method of Osteosynthesis, and Outcome in Radial Neck Fractures in Children: A Multicenter Study,” Journal of Pediatric Orthopaedics, Vol. 25, No. 1, 2005, pp. 45-50.

[13] F. A. Dawson and F. Inostroza, “Arthroscopic Reduction and Percutaneous Fixation of a Radial Neck Fracture in a Child,” Arthroscopy, Vol. 20, Suppl. 2, 2004, pp. 90-93. doi:10.1016/j.arthro.2004.04.013

[14] C. G. Nehr and M. A. Torch, "New Reduction Technique for Severely Displaced Pediatric Radial Neck Fractures," Journal of Pediatric Orthopaedics, Vol. 23, No. 5, 2003, pp. 626-628. doi:10.1097/01241398-200309000-00009

[15] S. M. Bernstein, P. McKeever and L. Bernstein, "Percutaneous Reduction of Displaced Radial Neck Fractures in Children," Journal of Pediatric Orthopaedics, Vol. 13, No. 1, 1993, pp. 85-88. doi:10.1097/01241398-199301000-00017

[16] J. V. Pesudo, J. Aracil and M. Barcelo, "Leverage Method in Displaced Fractures of the Radial Neck in Children," Clinical Orthopaedics and Related Research, Vol. 169, 1982, pp. 215-218.

[17] J. A. Steele and H. K. Graham, “Angulated Radial Neck Fractures in Children: A Prospective study of Percutaneous Reduction," The Journal of Bone and Joint Surgery (British Volume), Vol. 74-B, 1992, pp. 760-764.

[18] S. Yarar, D. W. Sommerfeldt, S. Gehrmann and J. M. Rueger, "Severely Displaced Radial Neck Fractures after Minimally Invasive Joystick Reduction and Prevot Nailing: Long-Term Course in Childhood," Unfallchirug, Vol. 110, No. 5, 2007, pp. 460-466. doi:10.1007/s00113-006-1181-7

[19] M. Ursei, J. Sales de Gauzy, J. Knorr, A. Abid, P. Darodes and J. P. Cahuzac, "Surgical Treatment of Radial Neck Fractures in Children by Intramedullary Pinning," Acta Orthopaedica Belgica, Vol. 72, No. 2, 2006, pp. 131-137.

[20] K. R. Prathapkumar, N. K. Garg and C. E. Bruce, "Elastic Stable Intramedullary Nail Fixation for Severely Displaced Fractures of the Neck of the Radius in Children," The Journal of Bone and Joint Surgery (British Volume), Vol. 88-B, No. 3, 2006, pp. 358-361. doi:10.1302/0301-620X.88B3.17099

[21] F. Garcia-Alvarez, J. Gil-Albarova, M. L. Bello, A. L. Bueno and F. Seral, "Results in Displaced Radial Neck Fractures in Children. Metaizeau Technique Versus Arthrotomy," Chir Organi Mov, Vol. 86, No. 3, 2001, pp. 211-217. 